Paper

\title{
Channel Estimation Scheme under Class A Impulsive Noise Environment
}

\author{
Hirotomo Yasui $^{\dagger}$, Akira Nakamura (member) ${ }^{\dagger}$, Kohei Ohno ${ }^{\dagger \dagger}$, \\ Makoto Itami (member) ${ }^{\dagger}$
}

\begin{abstract}
OFDM (Orthogonal Frequency Division Multiplexing) is the modulation scheme that is widely used in broad band power line communication (PLC). In OFDM transmission, impulsive noise is one of the major factors that limits the performance of PLC systems. In order to sufficiently reduce the influence of impulsive noise, precise estimation of channel transfer function is necessary. In this paper, the estimation scheme of channel transfer function under existence of additive impulsive noise is proposed. In the proposed scheme, successive two pilot OFDM symbols are used for reduction of the influence of impulsive noise. As the results, it is possible to perform precise estimation of channel transfer functions.
\end{abstract}

Key words: Channel Estimation, Impulsive Noise, Pilot Signal, Class A, OFDM, PLC.

\section{Introduction}

OFDM (Orthogonal Frequency Division Multiplexing) is a modulation scheme that is used in digital terrestrial television broadcasting, LTE (Long Term Evolution), PLC (Power Line Communication) and so on. OFDM is one of the multi-carrier modulation schemes and it transmits data using orthogonal sub-carriers. It is possible to use frequency band which is allocated to each system effectively because a large number of closely spaced orthogonal sub-carriers are used. It is possible to remove the influence of ISI (Inter-Symbol Interference) by adding the guard interval (GI) when guard interval length is longer than the delay spread of the transmission channel ${ }^{11}$.

In the PLC systems, reception characteristics become worse by the existence of the impulsive noise which is generated by the switching of various electric

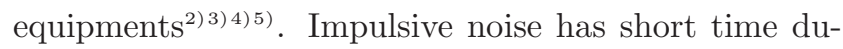
ration and very high magnitude. All sub-carriers of the OFDM symbols are severely influenced by impulsive noise because impulsive noise which is added to the OFDM symbol in time domain consists of broad frequency component. Therefore, many schemes such as clipping scheme ${ }^{4) 6}$, nulling scheme ${ }^{4) 6}$, clipping and nulling scheme ${ }^{2) 4}$, subtraction of estimated replica signal of impulsive noise ${ }^{7}$ are proposed to reduce the in-

Received September 3, 2015; Accepted October 6, 2015

$\dagger$ Department of Applied Electronics, Tokyo University of Science

(6-3-1 Nijyuku, Katsushikaku, Tokyo, 125-8585 Japan)

$\dagger \dagger$ Meiji University

(4-21-1 Nakano, Nakano,Tokyo, 164-8525 Japan) fluence of the impulsive noise in OFDM systems. In addition to these impulsive noise cancellation, it is necessary to perform accurate channel estimation by using the pilot symbols under impulsive noise environment in order to equalize the data symbols. Channel estimation schemes under impulsive noise environment in a variety of paper such $\mathrm{as}^{13)}$ has been proposed. In the above reference ${ }^{13)}$, in order to mitigate the impulsive noise, it is assumed that there are many OFDM symbols that contain no impulse burst. In our paper, the more general environment where multiple impulsive noises exist in one OFDM symbol is assumed. Pilot symbols that are used to estimate the channel transfer function are also influenced by impulsive noise. Therefore, it is necessary to reduce the influence of impulsive noise when channel transfer function is estimated.

In this paper, precise channel estimation scheme under impulsive noise environment is proposed. In the proposed scheme, successive two OFDM symbols are used for reduction of influence of impulsive noise.

This paper consists of the following sections. In section 2, OFDM transmission and the influence of impulsive noise on OFDM signal are briefly described. In section 3 , the conventional channel estimation scheme is described. In section 4 , the proposed channel estimation scheme is described. In these sections, the scheme to detect the impulsive noise on OFDM symbol using the pilot symbols is also described. The results of simulation are shown in section 5. Finally, this paper is concluded in Section 6 . 


\section{System Model}

In this section, the system model is explained. Fig. 1 shows the block diagram of the OFDM transmission system that is considered in this paper. In Fig. 1,

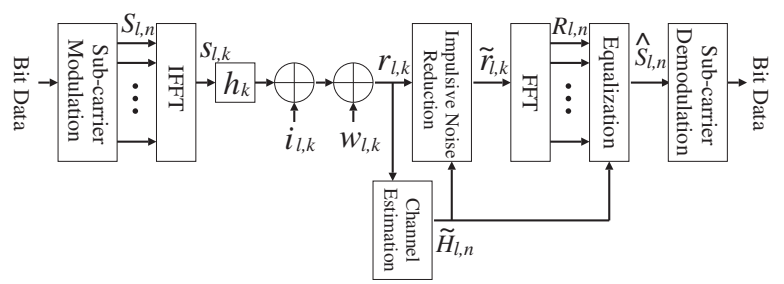

Fig. 1 Sytem Model

transmitted signal at time $t, s(t)$, is expressed as

$$
s(t)=\frac{1}{\sqrt{N}} \sum_{n=0}^{N-1} S_{l, n} e^{j 2 \pi\left(f_{c}+n f_{0}\right) t}
$$

where $N$ denotes the number of sub-carriers and $S_{l, n}$ denotes the complex data symbol transmitted in the $n$-th sub-carrier of $l$-th OFDM symbol. Sub-carrier spacing and lowest sub-carrier frequency are denoted by $f_{0}$ and $f_{c}$ respectively. If it is assumed that $s_{l, k}$ is generated by sampling $s(t)$ with the sampling frequency of $1 /\left(N f_{0}\right)$, received OFDM sample, $r_{l, k}$, which is affected by the multipath channel and additive noises is expressed as

$$
r_{l, k}=h_{k} \otimes s_{l, k}+w_{l, k}+i_{l, k} \quad(k=0,1, \ldots, N-1)(2)
$$

where $h_{k}$ denotes the impulse response of the transmission channel. In this paper, it is assumed that delay spread of the transmission channel is shorter than the guard interval length. $w_{l, k}$ denotes the additive white Gaussian noise and it is the random variable that follows the Gaussian distribution. $i_{l, k}$ denotes the additive impulsive noise that is added to the specific samples randomly. Generally, $i_{l, k}$ has a high magnitude and a random phase. By applying the fast Fourier transform (FFT) to $r_{l, k}$, the symbol transmitted on the $n$-th subcarrier in $l$-th OFDM symbol, $R_{l, n}$, is obtained by the following formula.

$$
\begin{aligned}
R_{l, n} & =\frac{1}{\sqrt{N}} \sum_{k=0}^{N-1} r_{l, k}\left(e^{-j \frac{2 \pi}{N}}\right)^{k n} \\
& =H_{n} S_{l, n}+W_{l, n}+\frac{1}{\sqrt{N}} \sum_{k=0}^{N-1} i_{l, k}\left(e^{-j \frac{2 \pi}{N}}\right)^{k n}(3)
\end{aligned}
$$

where $H_{n}$ denotes the channel transfer function corresponding to the $n$-th sub-carrier, $W_{l, n}$ denotes the complex Gaussian noise obtained by applying FFT to $w_{l, k}$, the third term in (3) denotes the influence of the impulsive noise against the $n$-th data symbol. In order to equalize $R_{l, n}$ in (3), it is necessary to estimate channel transfer function, $H_{n}$. In this paper, pilot symbols that are known by the receiver is used for channel estimation. Channel estimation is performed by dividing received OFDM symbol, $R_{l, n}$, by the pilot symbol, $S_{l, n}$, in the zero forcing equalizer as

$$
\begin{aligned}
\tilde{H}_{n} & =\frac{R_{l, n}}{S_{l, n}} \\
& =H_{n}+\frac{W_{l, n}}{S_{l, n}}+\frac{1}{S_{l, n} \sqrt{N}} \sum_{k=0}^{N-1} i_{l, k}\left(e^{-j \frac{2 \pi}{N}}\right)^{k n}(4)
\end{aligned}
$$

where $\tilde{H}_{n}$ denotes estimated channel transfer function. In (4), first term, $H_{n}$, denotes actual channel transfer function and the second term and later contain influence of additive noise. As shown in (4), influence of additive impulsive noise appears on whole symbol because the additive impulsive noise consists of broad frequency components. Accuracy of estimation is degraded by the impulsive noise whose magnitude is very large. Therefore, impulsive noise mitigation is also required to estimate channel transfer function precisely. In the next section, channel estimation scheme under existence of impulsive noise is described.

\section{Conventional Impulsive Noise Reduc- tion Techniques}

In this section, conventional channel estimation schemes are explained. Conventionally "clipping scheme" 4)6), "nulling scheme" 2)4), "clipping and nulling scheme" 2)4) have been proposed. In the following subsections, these schemes are explained.

\subsection{Clipping Scheme}

Clipping can be applied to the received signal, $r_{l, k}$, to reduce the influence of the impulsive noise by limiting the amplitude of the signal exceeding the threshold as shown in Fig. 2. Against the received signal, $r_{l, k}$,

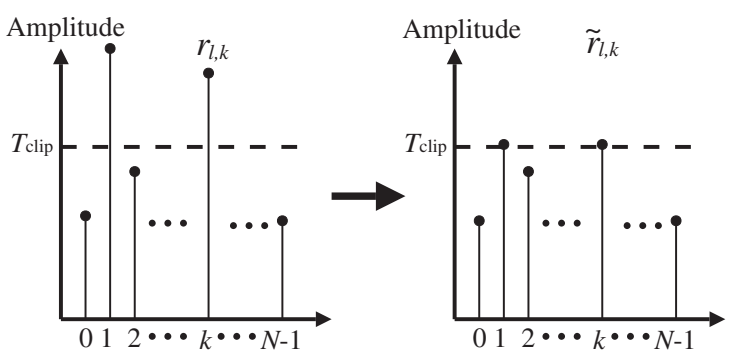

Fig. 2 Pattern Diagram of the Clipping Scheme

received signal after impulsive noise reduction, $\tilde{r}_{l, k}$, are given as 


$$
\tilde{r}_{l, k}= \begin{cases}r_{l, k} & \left(\left|r_{l, k}\right| \leqq T_{\text {clip }}\right) \\ T_{\text {clip }} e^{j \arg r_{l, k}} & \left(\left|r_{l, k}\right|>T_{\text {clip }}\right)\end{cases}
$$

where $T_{\text {clip }}$ denotes the clipping threshold that is defined $\mathrm{by}^{4) 6}$ )

$$
T_{\text {clip }}=2.2 \sigma_{s}
$$

where $\sigma_{s}$ denotes the standard deviation of OFDM signal.

\section{2 Nulling Scheme}

Nulling can be applied to the received signal, $r_{l, k}$, to reduce the influence of the impulsive noise by zeroing the amplitude of the signal exceeding the threshold as shown in Fig. 3. Against the received signal, $r_{l, k}$, re-

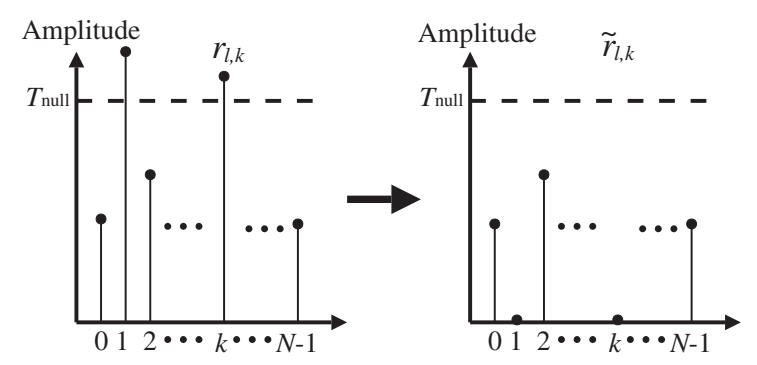

Fig. 3 Pattern Diagram of the Nulling Scheme

ceived signal after impulsive noise reduction, $\tilde{r}_{l, k}$, are given as

$$
\tilde{r}_{l, k}= \begin{cases}r_{l, k} & \left(\left|r_{l, k}\right| \leqq T_{\text {null }}\right) \\ 0 & \left(\left|r_{l, k}\right|>T_{\text {null }}\right)\end{cases}
$$

where $T_{\text {null }}$ denotes the nulling threshold that is defined $\mathrm{by}^{4) 6) 3)}$

$$
T_{\text {null }}=3.08 \sigma_{s}
$$

\section{3 Clipping and Nulling Scheme}

Clipping and nulling ${ }^{2(4)}$ is the scheme which combines clipping and nulling schemes as shown Fig. 4. Against

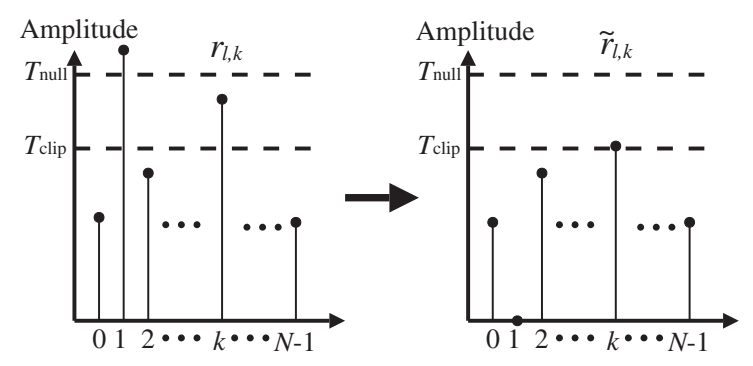

Fig. 4 Pattern Diagram of the Clipping and Nulling Scheme

the received signal, $r_{l, k}$, received signal after impulsive noise reduction, $\tilde{r}_{l, k}$, are given as

$$
\tilde{r}_{l, k}= \begin{cases}r_{l, k} & \left(\left|r_{l, k}\right| \leqq T_{\text {clip }}\right) \\ T_{\text {clip }} e^{j \arg r_{l, k}} & \left(T_{\text {clip }}<\left|r_{l, k}\right| \leqq T_{\text {null }}\right) \\ 0 & \left(\left|r_{l, k}\right|>T_{\text {null }}\right)\end{cases}
$$

\section{Proposed Channel Estimation Scheme}

In this section, proposed channel estimation scheme is described. The proposed channel estimation is performed using the known pilot symbols. Fig. 5 shows the allocation of pilot symbols in the proposed scheme. In the proposed scheme, the pilot symbols allocated in

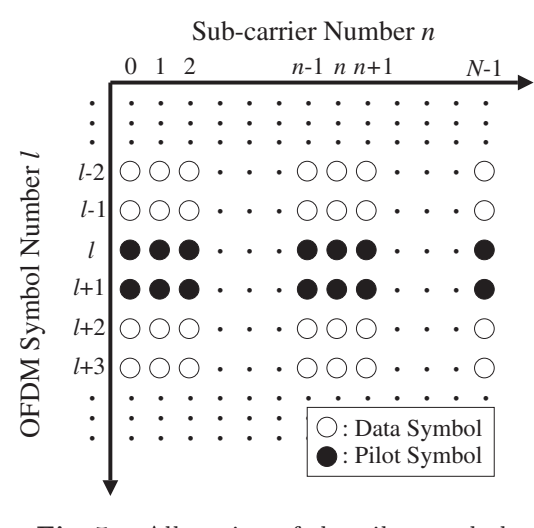

Fig. 5 Allocation of the pilot symbols

the two successive OFDM symbols are used for channel estimation. If there is no impulsive noise, the channel transfer function can easily be estimated. However, under the impulsive noise environment, more channel estimation error occurs because of additive impulsive noise in the received pilot symbols. If only one OFDM symbol that contains pilot symbols is used for channel estimation and impulsive noise reduction, it is very complex to separate the influences of the channel transfer function and the additive impulsive noise. Therefore, the temporally consecutive two OFDM symbols are used. The pilot symbols are BPSK (Binary Phase Shift Keying) modulated symbols and different patterns are used in the $l$ th and $(l+1)$ th OFDM symbols. The procedure for channel estimation reducing the impulsive noise is shown in Fig. 6. In the proposed scheme, at first, FFT is applied two time domain samples to perform coarse channel estimation against each OFDM symbol in the zero forcing equalizer. If it is assumed that $S_{l, n}$ and $S_{l+1, n}$ are the pilot symbols that are allocated in the $l$ th and $(l+1)$ th OFDM symbols respectively, the coarsely estimated channel transfer functions corresponding to the $l$ th and $(l+1)$ th OFDM symbols, $\tilde{H}_{l, n}^{(j)}$ and $\tilde{H}_{l+1, n}^{(j)}$, are obtained in the following formulas.

$$
\tilde{H}_{l, n}^{(j)}=\frac{R_{l, n}^{(j)}}{S_{l, n}}
$$




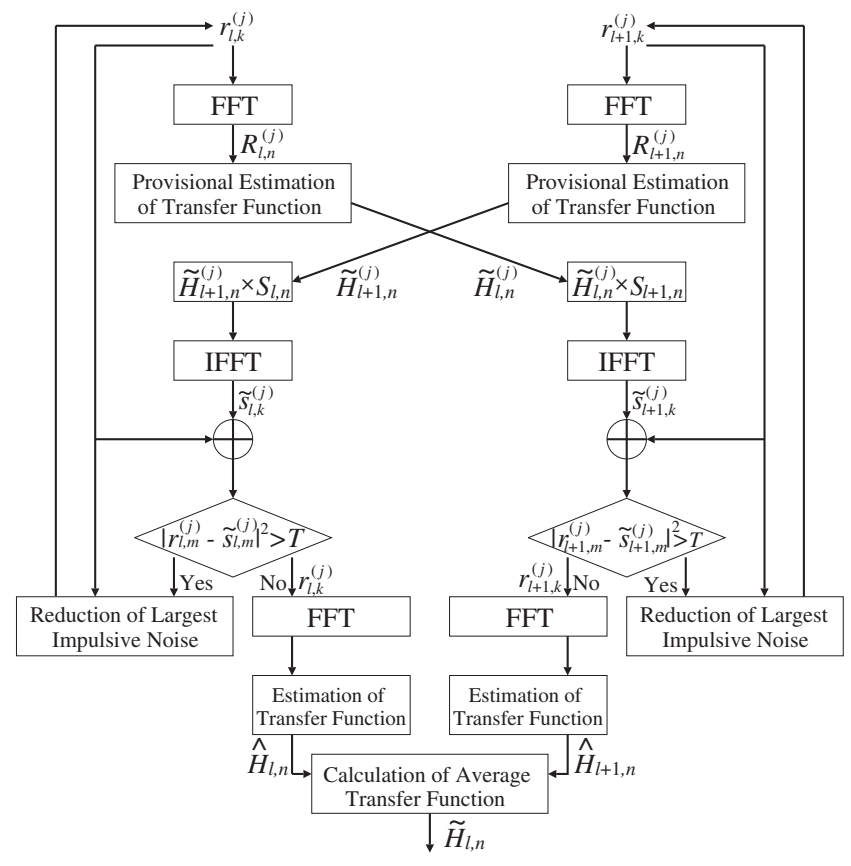

Fig. 6 The block diagram of the proposed channel estimation scheme

$$
\begin{aligned}
& =H_{n}+\frac{W_{l, n}}{S_{l, n}} \\
& +\frac{1}{S_{l, n} \sqrt{N}} \sum_{k=0}^{N-1} i_{l, k}\left(e^{-j \frac{2 \pi}{N}}\right)^{k n}
\end{aligned}
$$

$$
\begin{aligned}
\tilde{H}_{l+1, n}^{(j)} & =\frac{R_{l+1, n}^{(j)}}{S_{l+1, n}} \\
& =H_{n}+\frac{W_{l+1, n}}{S_{l+1, n}} \\
& +\frac{1}{S_{l+1, n} \sqrt{N}} \sum_{k=0}^{N-1} i_{l+1, k}\left(e^{-j \frac{2 \pi}{N}}\right)^{k n}
\end{aligned}
$$

Where $H_{n}$ denotes the actual channel transfer function and where $j$ denotes iteration count. In the proposed scheme, the time domain OFDM samples against the $l$ th OFDM symbol, $\tilde{s}_{l, k}^{(j)}$, is re-generated using the pilot symbols assigned to the $l$ th OFDM symbol, $S_{l, n}$, and the channel transfer function estimated using the $(l+1)$ th OFDM symbol, $\tilde{H}_{l+1, n}^{(j)}$, as shown in the following formula.

$$
\begin{aligned}
\tilde{s}_{l, k}^{(j)} & =\frac{1}{\sqrt{N}} \sum_{n=0}^{N-1} \tilde{H}_{l+1, n}^{(j)} S_{l, n}\left(e^{-j \frac{2 \pi}{N}}\right)^{k n} \\
& =h_{k} \otimes s_{l, k}+\frac{1}{\sqrt{N}} \sum_{n=0}^{N-1} \frac{S_{l, n}}{S_{l+1, n}} W_{l, n}\left(e^{-j \frac{2 \pi}{N}}\right)^{k n} \\
& +\frac{1}{N} \sum_{q=0}^{N-1} i_{l, k} \sum_{k=0}^{N-1} \frac{S_{l, n}}{S_{l+1, n}}\left(e^{-j \frac{2 \pi}{N}}\right)^{(k-q) n}
\end{aligned}
$$

In this case, since the pilot symbol patterns are different in the $l$ th and $(l+1)$ th OFDM symbols, the peak of impulsive noise added to the $l$ th OFDM symbol is spread over time domain and reduced by using $\tilde{H}_{l+1, n}^{(j)}$ for re-generation as expressed by the third term in (12). Therefore, by calculating difference signal, $\left|r_{l, k}^{(j)}-\tilde{s}_{l, k}^{(j)}\right|^{2}$, between the received time domain samples corresponding to the $l$ th OFDM symbol, $r_{l, k}^{(j)}$, and this re-generated time domain samples, $\tilde{s}_{l, k}^{(j)}$, it is possible to detect the impulsive noises that were added to the $l$ th OFDM symbols using the pre-defined threshold value, $T$. The threshold value, $T$, in (13) derived in the reference ${ }^{7)}$ is used to detect additive impulsive noise in this paper.

$$
T \equiv 0.06 \cdot\left(2 \sigma^{2}\right)
$$

where $\sigma^{2}$ denotes the average power of the additive noise. Sample, $r_{l, m}^{(j)}$, which is the largest difference signal, $\left|r_{l, m}^{(j)}-\tilde{s}_{l, m}^{(j)}\right|^{2}$, among the difference signal, $\mid r_{l, k}^{(j)}-$ $\left.\tilde{s}_{l, k}^{(j)}\right|^{2}$, exceeding the threshold, $T$, is replaced by the re-generated sample, $\tilde{s}_{l, m}^{(j)}$, as

$$
r_{l, m}^{(j+1)}=\tilde{s}_{l, m}^{(j)} \text { if }\left|r_{l, m}^{(j)}-\tilde{s}_{l, m}^{(j)}\right|^{2}>T
$$

where $m$ denotes the sample number of the largest difference signal, $\left|r_{l, k}^{(j)}-\tilde{s}_{l, k}^{(j)}\right|^{2}$, in other words, $m$ is the time sample number where the largest amplitude impulsive noise is added. After that, the above process is repeated $j$ times until all the samples are below the threshold value, $T$, as shown in Fig. 6 .

The same procedure is performed again the $(l+1)$ th OFDM symbol using $\tilde{H}_{l, n}^{(j)}$ and the pilot symbols allocated to the $(l+1)$ th OFDM symbol, $S_{l+1, n}$ as shown in the following formula.

$$
\begin{aligned}
\tilde{s}_{l+1, k}^{(j)} & =\frac{1}{\sqrt{N}} \sum_{n=0}^{N-1} \tilde{H}_{l, n}^{(j)} S_{l+1, n}\left(e^{-j \frac{2 \pi}{N}}\right)^{k n} \\
& =h_{k} \otimes s_{l+1, k}+\frac{1}{\sqrt{N}} \sum_{n=0}^{N-1} \frac{S_{l+1, n}}{S_{l, n}} W_{l+1, n}\left(e^{-j \frac{2 \pi}{N}}\right)^{k n} \\
& +\frac{1}{N} \sum_{q=0}^{N-1} i_{l+1, k} \sum_{k=0}^{N-1} \frac{S_{l+1, n}}{S_{l, n}}\left(e^{-j \frac{2 \pi}{N}}\right)^{(k-q) n}
\end{aligned}
$$

In this case, since the pilot symbol patterns are different in the $l$ th and $(l+1)$ th OFDM symbols, the peak of impulsive noise added to the $(l+1)$ th OFDM symbol is spread over time domain and reduced by using $\tilde{H}_{l, n}^{(j)}$ for re-generation as expressed by the third term in (15). Therefore, by calculating difference signal, $\left|r_{l+1, k}^{(j)}-\tilde{s}_{l+1, k}^{(j)}\right|^{2}$, between the received time domain samples corresponding to the $(l+1)$ th OFDM symbol, $r_{l+1, k}^{(j)}$, and this re-generated time domain samples, $\tilde{s}_{l+1, k}^{(j)}$, it is possible to detect the impulsive noises that were added to the $(l+1)$ th OFDM symbols using the 
pre-defined threshold value, $T$. Sample, $r_{l+1, m}^{(j)}$, which is the largest difference signal, $\left|r_{l+1, m}^{(j)}-\tilde{s}_{l+1, m}^{(j)}\right|^{2}$, among the difference signal, $\left|r_{l+1, k}^{(j)}-\tilde{s}_{l+1, k}^{(j)}\right|^{2}$, exceeding the threshold, $T$, is replaced by the re-generated sample, $\tilde{s}_{l+1, m}^{(j)}$, as

$$
r_{l+1, m}^{(j+1)}=\tilde{s}_{l+1, m}^{(j)} \text { if }\left|r_{l+1, m}^{(j)}-\tilde{s}_{l+1, m}^{(j)}\right|^{2}>T
$$

The above process is repeated $j$ times alternately between $l$ th and $(l+1)$ th OFDM symbols until all the samples are below the threshold value, $T$, as shown Fig. 6. As the result, the influence of impulsive noise can be reduced from both $l$ th and $(l+1)$ th OFDM symbols. After reducing the influence of the impulsive noise, channel estimation is performed again to obtain more precise channel transfer functions, $\hat{H}_{l, n}, \hat{H}_{l+1, n}$ in the zero forcing equalizer. By using updated transfer functions, further impulsive noise reduction and channel estimation is performed iteratively until the impulsive noise beyond the threshold value is not detected. After this, the average value of the transfer functions estimated by the $l$ th and $(l+1)$ th OFDM symbols, $\tilde{H}_{n}$, are used for zero forcing equalization and impulsive noise reduction for following data symbols.

\section{Results of Simulation}

In this section, the accuracy of the conventional and proposed schemes are evaluated and bit error rate characteristics of the proposed scheme are also evaluated by the computer simulation. It is assumed that channel characteristics are time-invariant.

\subsection{Impulsive Noise Model}

In this paper, Middleton's Class A impulsive noise model $^{8)}$ is used to evaluate the accuracy of channel estimation by the computer simulations. This is a model which represents the Gaussian noise and impulsive noise simultaneously. It is known that Class A noise well expresses the noise generated in the actual PLC systems. Probability density function of Middleton's Class A noise model is shown as

$$
\begin{aligned}
p(x) & =\sum_{u=0}^{\infty} \frac{e^{-A} A^{u}}{u !} \cdot \frac{1}{\sqrt{2 \pi} \sigma_{u}} \exp \left(-\frac{|x|^{2}}{2 \sigma_{u}^{2}}\right) \\
& =\sum_{u=0}^{\infty} P_{P, A}(u) \cdot p_{G, 0, \sigma_{u}^{2}}(x)
\end{aligned}
$$

where $A$ denotes the probability where the impulsive noise is added to each sample, $P_{P, A}(u)=e^{-A} A^{u} / u$ ! denotes the probability density function of the Poisson distribution whose average value is $A \cdot p_{G, 0, \sigma_{u}^{2}(x)}=$ $\frac{1}{\sqrt{2 \pi} \sigma_{u}} \exp \left(-\frac{|x|^{2}}{2 \sigma_{u}^{2}}\right)$ is the probability density function of the Gaussian distribution whose average value and variance are 0 and $\sigma_{u}^{2}$ respectively. If it is assumed that the number of impulsive noise sorces is denoted by $u, \sigma_{u}^{2}$ is expressed as

$$
\sigma_{u}^{2}=\frac{\sigma^{2}\left(\frac{u}{A}+\Gamma\right)}{1+\Gamma}=u \cdot \frac{\sigma_{I}^{2}}{A}+\sigma_{W}^{2}
$$

where $\Gamma=\sigma_{W}^{2} / \sigma_{I}^{2}$ denotes the ratio of the power of the Gaussian noise, $\sigma_{W}^{2}$, against the average power of the impulsive noise, $\sigma_{I}^{2}$. Total noise, $\sigma^{2}$, is defined by $\sigma_{W}^{2}+\sigma_{I}^{2}$.

\section{2 Comparison of Estimation Error}

In this section, the estimation error of the proposed scheme is compared to those of conventional schemes. Parameters used in the simulation for channel estimation are shown in Table 1. In this paper, the values of $A$ and $\Gamma$ that are introduced in the previous researches ${ }^{\text {7)1011)12) }}$ are used. Accuracy of estimation is

Table 1 SIMULATION PARAMETERS

\begin{tabular}{lcl}
\hline \hline Number of sub-carriers $(N)$ & $:$ & 1024 \\
Modulation scheme of pilot sub-carriers & $:$ & BPSK \\
Number of OFDM symbols & $:$ & 1000 \\
Carrier interval $\left(f_{0}\right)$ & $:$ & $20.0[\mathrm{kHz}]$ \\
Lowest sub-carrier frequency $\left(f_{c}\right)$ & $:$ & $2.0[\mathrm{MHz}]$ \\
Channel model & $:$ & Two-path model \\
DUR(Desired to Undesired Ratio $)$ & $:$ & $10[\mathrm{~dB}]$ \\
Delay time of second path & $:$ & $3.0[\mu \mathrm{s}]$ \\
Impulse index $(A)$ & $:$ & 0.01 \\
Impulse to Gaussian noise ratio $(\Gamma)$ & $:$ & 0.01 \\
\hline \hline
\end{tabular}

evaluated by RMSE (root mean square error). RMSE between the actual transfer function, $H_{n}$, and the estimated transfer function, $\tilde{H}_{n}$, is defined by

$$
R M S E=\sqrt{\frac{\sum_{n=0}^{N-1}\left(\tilde{H}_{n}-H_{n}\right)^{2}}{\sum_{n=0}^{N-1} H_{n}^{2}}}
$$

RMSE characteristics against CNR (Carrier to Noise Ratio) of the conventional scheme and the proposed scheme are shown in Fig. 7. In Fig.7, "Gaussian Noise Only" denotes additive noise is only Gaussian noise without impulsive noise. In the following legends, Class A noise is used for the computer simulation. "No Reduction" denotes the reduction of impulsive noise is not performed. "Clipping", "Nulling", "Clipping and Nulling" and "Proposed Scheme" denotes clipping scheme, nulling scheme, clipping and nulling scheme and proposed scheme are respectively performed to the reduction of the additive impulsive noise. In the clipping scheme as shown in Fig. 7, there is a floor in RMSE characteristic because not only additive noise but also 


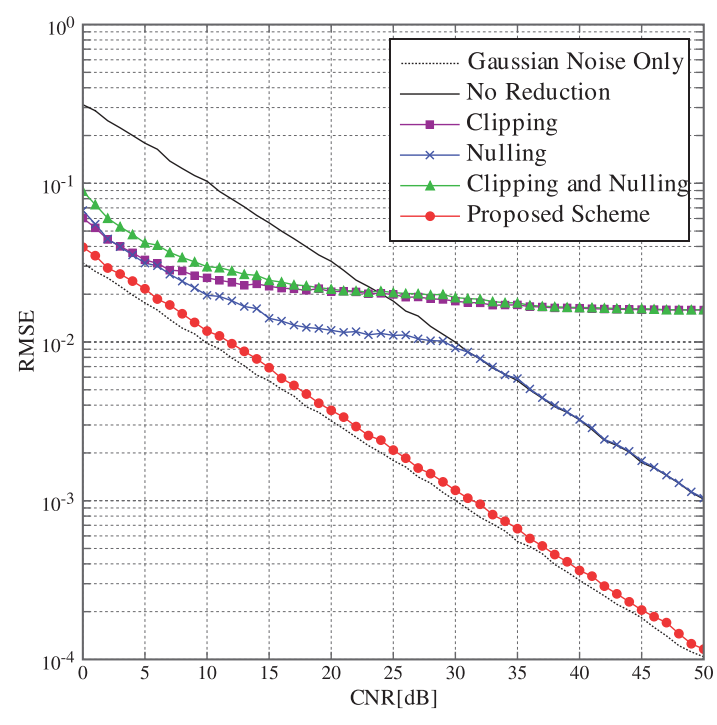

Fig. 7 RMSE characteristics against CNR

OFDM signal are clipped and performance degrades worse than the case where no reduction is performed. In the nulling scheme, RMSE is not well improved and is almost equivalent to the case where no reduction is performed under high CNR environment because the optimal threshold value in nulling scheme is larger than that in clipping scheme and the small additive impulsive noise is not detected. The RMSE of the clipping and nulling scheme is slightly better than that of the clipping scheme and is almost equivalent to that of the clipping scheme under high CNR environment.

On the other hand, proposed scheme can achieve the best performance than the conventional schemes. Therefore, the proposed channel estimation scheme is effective under impulsive channel environments. In the next subsection, evaluation of the bit error rate characteristics is performed using the estimated transfer function.

\section{3 Bit Error Rate Characteristics}

In this paper, time sample replacement scheme ${ }^{9)}$ is used for reduction of impulsive noise on the data symbol. The details of this scheme is shown in the referenced paper ${ }^{9)}$.

Fig. 8 shows the block diagram of time sample replacement scheme. The signal after zero forcing equalization, $\hat{S}_{n}$, is remapped as shown in Fig. 9.

Time domain sample, $\tilde{s}_{k}$, is re-generated by performing IFFT to the $H_{n} \tilde{S}_{n}$. If $\left|r_{m}-\tilde{s}_{m}\right|^{2}>T, r_{m}$ is replaced by $\tilde{s}_{m}$ where $m$ denotes time sample number that maximizes the difference signal, where $T$ denotes the threshold value defined as (13). The above procedure is iteratively performed until all sample values of

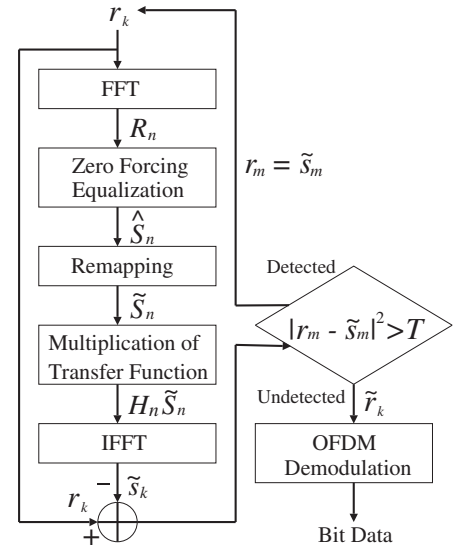

Fig. 8 The block diagram of the impulsive noise canceller

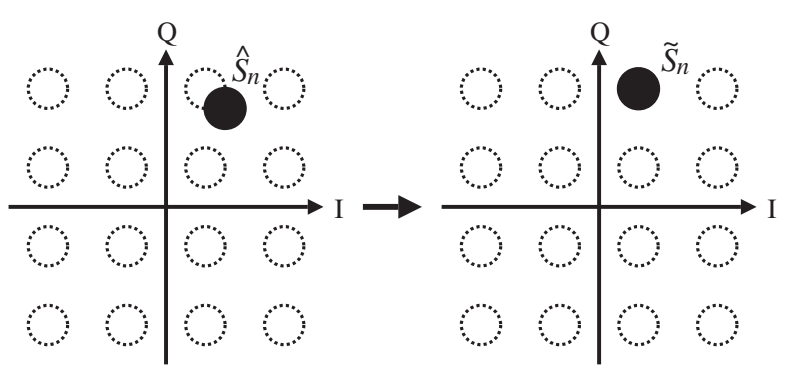

Fig. 9 Remapping Process

the difference signal are below the threshold value, $T$.

Modulation schemes of the data symbol are assumed to be QPSK and 16QAM. In all legends, Class A noise

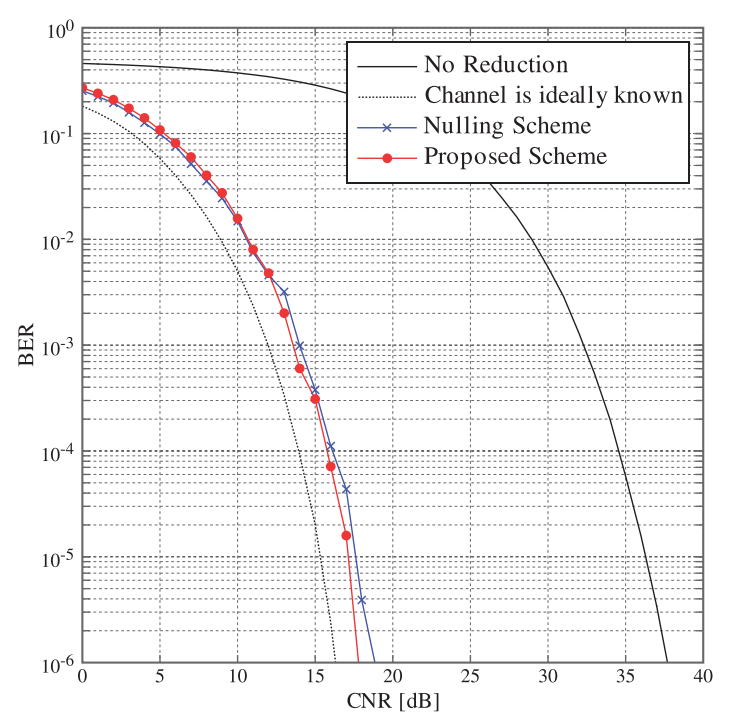

Fig. 10 BER characteristics against CNR (QPSK)

is used for additive noise. "No Redection" denotes the reduction of impulsive noise is not performed. In other legends, time sample replacement scheme is used for reducing the influence of impulsive noise on the data symbol. "Channel is ideally known" denotes that channel transfer function, $H_{n}$, is ideally known in the re- 


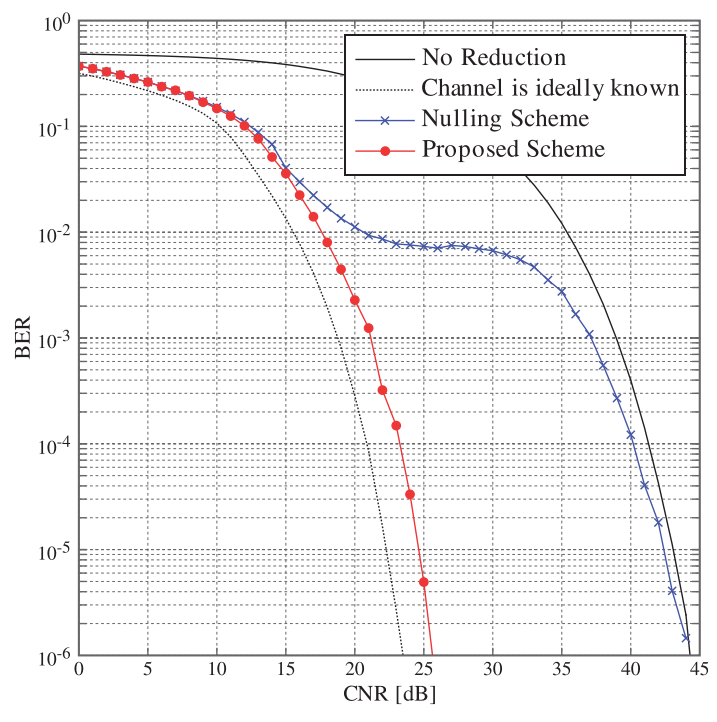

Fig. 11 BER characteristics against CNR (16QAM)

ceiver. "Nulling Scheme" and "Proposed Scheme" denotes nulling scheme and proposed scheme are respectively performed to the reduction of the additive impulsive noise for channel estimation. In the QPSK case in Fig.10, performances of the proposed and conventional schemes are almost equivalent because the sensitivity of QPSK detection is considered to be less sensitive against the channel estimation error. However, in 16QAM cases in Fig. 11, the performances of the conventional scheme much degrades as compared to the proposed scheme. This is because the sensitivity of the conventional scheme against channel estimation error is larger for the high constellation modulation scheme. On the other hand, the proposed scheme can achieve the performance almost equivalent to the QPSK case even in the 16QAM case. As shown in the above results, the proposed channel estimation scheme can achieve more precise channel estimation than the conventional schemes.

\section{Conclusion}

In this paper, the channel estimation scheme under the impulsive PLC channel was proposed. The performance of the proposed scheme is compared to the channel estimation schemes using the clipping and nulling under the Middleton's Class A noise environment. As the result of simulations, RMSE of the estimated channel transfer function in the proposed scheme can be most improved. Moreover, the BER performances against data symbols using the estimated channel were also compared and it was confirmed that the proposed scheme can achieve better performance in the high rate modulation schemes such as 16QAM.

In the further researches, theoretical optimization of the proposed scheme and complexity reduction are studied.

\section{References}

1) Fatma Abdelkefi, Abraham Gabay and Pierre Duhamel : "Impulse Noise Cancellation in Multicarrier Transmission", IEEE Int. Conf. on International Conference of Acoustics, Speech and Signal Processing (ICASSP), vol.4, pp.2381-2384 (May 2001)

2) Anil Mengi and A. J. Han Vinck: "Successive Impulsive Noise Suppression in OFDM", IEEE International Symposium on Power Line Communications and Its Applications (ISPLC), pp. 33-37 (Mar. 2010)

3) Sergey. V. Zhidkov: "Performance Analysis and Optimization of OFDM Receiver With Blanking Nonlinearity in Impulsive Noise Environment", IEEE Transactions on Vehicular Technology, 55 1, pp.234-242 (Jan. 2006)

4) Sergey. V. Zhidkov: "Analysis and Comparison of Several Simple Impulsive Noise Mitigation Schemes for OFDM Receivers", IEEE Transactions on Communications, 56, 1, pp.5-9 (Jan. 2008)

5) Homayoun Nikookar, Danesh Nathoeni: "Performance Evaluation of OFDM Transmission Over Impulsive Noisy Channels", IEEE International Symposium on Personal, Indoor and Mobile Radio Communications (PIMRC), 2, pp.550-554 (Sept. 2002)

6) H. A. Suraweera, C. Chai, J. Shentu, J. Amstrong: "Analysis of Impulsive Noise Mitigation Techniques for Digital Terevision Systems", in Proc. 8th Int. OFDM Workshop (InOWo'03), Hamburg, Germany, pp.172-176. (Sept. 2003)

7) Takuya Kitamura, Hiroshi Ando, Kohei Ohno, Makoto Itami: "The Impulsive Noise Reduction Using It's Replica Signal Under Class-A Impulsive Channel", WPMC2012 Conference, pp.460464, (Sept. 2012)

8) D. Middleton: "Procedures for determining the parameters of the first order canonical models of Class A and Class B electromagnetic interference", IEEE Trans. Electromagn. Compat., Vol. EMC-21, No.3, pp.190-208, (Aug. 1979)

9) Tatsuya Hirakawa, Masahiro Fujii, Makoto Itami, Kohji Ito: "Improving Influence of Impulse Noise to OFDM Signal by Recovering Time Domain Samples", Proc. of ICCE2006, pp.327-328, (Jan. 2006)

10) Stephen W. Lai and Geoffrey G. Messier: "The Wireless/Powerline Diversity Channel", IEEE ICC2010 Conference, pp.1-5, (May 2010)

11) Yu-Zhong Jiang, Xiu-lin $\mathrm{Hu}, \mathrm{Xu}$ Kai, and Zhai Qi: "Bayesian Estimation of Class A Noise Parameters with Hidden Channel States", IEEE ISPLC2007 Conference, pp.2-4, (Mar. 2007)

12) Hiroki Nakagawa, Daisuke Umehara, Satoshi Denno, and Yoshiteru Morihiro: "A Decoding for Low Density Parity Check Codes over Impulsive Noise Channels", ISPLC2007 Conference, pp.85-89, (Apr. 2005)

13) J. Rinne, A. Hazmi, M. Renfors: "Impulse burst position detection and channel estimation schemes for OFDM Systems", IEEE Trans. on Consumer Electronics, Vol. 49, No.3, pp.539-545, (Aug. 2003)

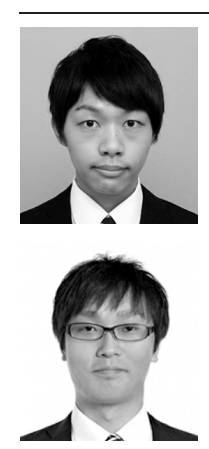

Hirotomo Yasui was born in Chiba, Japan in 1991. He graduated from Department of Applied Electronics, Tokyo University of Science in March, 2014. He is being enrolled in the Tokyo University of Science graduate school master course at present. $\mathrm{He}$ is engaged in the research of OFDM. He is a member of IEEE.

Akira Nakamura received his B.E. and M.E. degree in Electronics Engineering from Tokyo University of Science, Japan, in 2009 and 2011, respectively. He received Ph.D. degree of Electronics Engineering from Tokyo University of Science in 2013. He is an assistant professor at Dept. of Applied Electronics, Tokyo University of Science. His current research interests include wireless communication and broadcasting using OFDM modulation. He is a member of ITE, IEICE and IEEE. 
Kohei Ohno was born in 1980 in Tokyo, Japan. He received the B.E. M.E. and Dr. E. degrees in electrical engineering from Meiji University, Kanagawa, Japan, in 2002, 2004, and 2008 respectively. From 2008 to 2013, he was an assistant professor at Tokyo University of Science. Since 2013, he has been a senior assistant professor of Meiji University. His major interest includes UWB radio systems and spread spectrum technique. Dr. Ohno is a member of IEICE and IEEE.

Makoto Itami received B. Eng., M. Eng. and Dr Eng. degrees from the University of Tokyo, in 1984,1986 and 1989, respectively. In 1989, he joined the Department of Applied Electronics at Tokyo University of Science, Japan, and since 2008 he has been a Professor. His research interests are in the fields of communication systems and digital signal processing. He is a member of ITE, IEICE and IEEE. 